\title{
Images of Gaussian random fields: Salem sets and interior points
}

\author{
by \\ Narn-Ruein Shieh (Taipei) and Yimin Xiao (East Lansing, MI)
}

\begin{abstract}
Let $X=\left\{X(t), t \in \mathbb{R}^{N}\right\}$ be a Gaussian random field in $\mathbb{R}^{d}$ with stationary increments. For any Borel set $E \subset \mathbb{R}^{N}$, we provide sufficient conditions for the image $X(E)$ to be a Salem set or to have interior points by studying the asymptotic properties of the Fourier transform of the occupation measure of $X$ and the continuity of the local times of $X$ on $E$, respectively. Our results extend and improve the previous theorems of Pitt [24] and Kahane $[12,13]$ for fractional Brownian motion.
\end{abstract}

1. Introduction. Let $X=\left\{X(t), t \in \mathbb{R}^{N}\right\}$ be a centered Gaussian random field with values in $\mathbb{R}^{d}$. For a Borel set $E \subseteq \mathbb{R}^{N}$, its image $X(E)$ under $X$ is a random set in $\mathbb{R}^{d}$. Due to its connections to random fractals and harmonic analysis, it has been of interest to study the geometric and arithmetic properties of $X(E)$. We refer to Adler [1], Kahane [12], Khoshnevisan [20] for extensive studies of various properties of $X(E)$, where $X$ is either a fractional Brownian motion or the Brownian sheet.

When $X$ is an $(N, d)$-fractional Brownian motion of index $\alpha \in(0,1)$, then it is known that

$$
\operatorname{dim}_{H} X(E)=\min \left\{d, \frac{\operatorname{dim}_{H} E}{\alpha}\right\} \quad \text { a.s., }
$$

where $\operatorname{dim}_{\mathrm{H}}$ denotes Hausdorff dimension. Clearly, two distinct cases come up in (1.1): $\operatorname{dim}_{H} E>\alpha d$ or $\operatorname{dim}_{H} E \leq \alpha d$. In the first case, a Fourieranalytic argument due to Kahane (see [12]) shows that $X(E)$ a.s. has positive $d$-dimensional Lebesgue measure. The natural question whether $X(E)$ has non-empty interior was first studied by Kaufman [17] for $X$ being the standard Brownian motion in $\mathbb{R}$. Pitt [24] and Kahane [12], [13] ex-

2000 Mathematics Subject Classification: Primary 60G15, 60G17; Secondary 60G60, 42B10, 43A46, 28A80.

Key words and phrases: Gaussian random fields, fractional Brownian motion, fractional Riesz-Bessel motion, image, Hausdorff dimension, Fourier dimension, Salem set, local times.

Research of the second-named author partially supported by NSF grant DMS-0404729. 
tended Kaufman's result to fractional Brownian motion and proved that $X(E)$ a.s. has interior points provided $\operatorname{dim}_{\mathrm{H}} E>\alpha d$. In the case when $\operatorname{dim}_{\mathrm{H}} E \leq \alpha d$, Kahane [12], [13] has shown that $X(E)$ is a.s. a Salem set. That is, $\operatorname{dim}_{\mathrm{H}} X(E)=\operatorname{dim}_{\mathrm{F}} X(E)$ a.s., where $\operatorname{dim}_{\mathrm{F}}$ is Fourier dimension (see Section 2 for the definition).

This paper is concerned with the geometric and arithmetic properties of the image $X(E)$ for a large class of $(N, d)$-Gaussian random fields including fractional Brownian motion, fractional Riesz-Bessel motion (cf. Anh et al. [2]) and some Gaussian processes with stationary increments and discrete spectrum measures (cf. Xiao [28]). We should also mention that the methods in this paper can be modified to study the images of more general Gaussian random fields than those considered here. For example, similar results have been proven for certain anisotropic Gaussian random fields such as fractional Brownian sheets by $\mathrm{Wu}$ and Xiao [27].

Let $\mu$ be a probability measure carried by $E$ and let $\nu$ be the image measure of $\mu$ under the mapping $t \mapsto X(t)$. In Section 2 we study the asymptotic properties of the Fourier transform $\widehat{\nu}(\xi)$ as $\xi \rightarrow \infty$. Our observation is that, for a Gaussian random field $X$ with stationary increments, the asymptotic property of $\widehat{\nu}(\xi)$ is determined by the asymptotic behavior of the spectral measure of $X$ at infinity; see Section 2 for more information. Moreover, we show that, under certain conditions, the image $X(E)$ is a Salem set. These results extend those of Kahane [12], [13] to a large class of Gaussian random fields.

In Section 3 we prove a sufficient condition for $X(E)$ to have interior points. Even though we follow the approach of Pitt [24] and Kahane [12], [13] to reduce the problem of interior points to proving the existence of a continuous local time of $X$ on $E$, our method is finer than those used by the previous authors, and is more reminiscent of those in Cuzick and DuPreez [9] and Xiao and Zhang [32]. As a result, when $X$ is a fractional Brownian motion, our Theorem 3.2 improves the results of Pitt [24] and Kahane [12], [13].

There are two open questions that cannot be answered by the methods known to us, and we list them at the end of Sections 2 and 3, respectively.

Throughout this paper, we use $\langle\cdot, \cdot\rangle$ and $|\cdot|$ to denote the ordinary scalar product and the Euclidean norm in $\mathbb{R}^{m}$ respectively, no matter what the value of the integer $m$. Unspecified positive and finite constants will be denoted by $K$, which may have different values from line to line. Specific constants in Section $i$ will be denoted by $K_{i, 1}, K_{i, 2}$ and so on.

2. Salem sets. We start by recalling from Kahane [12], [13] the definitions of Fourier dimension and Salem set. Given a constant $\beta \geq 0$, a Borel set $F \subset \mathbb{R}^{d}$ is said to be an $M_{\beta}$ set if there exists a probability measure $\nu$ 
on $F$ such that

$$
|\widehat{\nu}(\xi)|=o\left(|\xi|^{-\beta}\right) \quad \text { as } \xi \rightarrow \infty
$$

Note that if $\beta>d / 2$, then (2.1) implies that $\widehat{\nu} \in L^{2}\left(\mathbb{R}^{d}\right)$ and, consequently, $F$ has positive $d$-dimensional Lebesgue measure. For any Borel set $F \subset \mathbb{R}^{d}$, its Fourier dimension $\operatorname{dim}_{\mathrm{F}} F$ is defined by

$$
\operatorname{dim}_{\mathrm{F}} F=\sup \left\{\alpha \in[0, d]: F \text { is an } M_{\alpha / 2} \text { set }\right\} .
$$

It follows from Frostman's theorem and the following formula for the $\alpha$ energy $(0<\alpha<d)$ of $\nu$ :

$$
I_{\alpha}(\nu)=K(\alpha, d) \int_{\mathbb{R}^{d}}|\widehat{\nu}(\xi)|^{2}|\xi|^{\alpha-d} d \xi,
$$

where $K(\alpha, d)>0$ is a constant depending on $\alpha$ and $d$ only (cf. Kahane $[12$, Ch. 10]), that $\operatorname{dim}_{F} F \leq \operatorname{dim}_{\mathrm{H}} F$. Strict inequality may hold. For example, the Fourier dimension of the triadic Cantor set is 0 , but its Hausdorff dimension is $\log 2 / \log 3$. Unlike the Hausdorff dimension $\operatorname{dim}_{\mathrm{H}} F$ which describes a metric property of $F$, the Fourier dimension $\operatorname{dim}_{\mathrm{F}} F$ is closely related to the arithmetic properties of $F$. As a further example of this aspect, we mention that every set $F \subset \mathbb{R}^{d}$ with positive Fourier dimension generates $\mathbb{R}^{d}$ as a group.

We say that a Borel set $F$ is a Salem set if $\operatorname{dim}_{\mathrm{F}} F=\operatorname{dim}_{\mathrm{H}} F$. Such sets are of importance in studying the problem of uniqueness and multiplicity for trigonometric series (cf. Zygmund [34] and Kahane and Salem [15]) and the restriction problem for the Fourier transforms (cf. Mockenhaupt [22]). The first construction of such a set $F$ on the line with $0<\operatorname{dim}_{\mathrm{F}} F<1$ was given by Salem in 1950 (cf. Kahane and Salem [15, Chapter 8]) using probabilistic methods. Kahane [12], [13] has constructed Salem sets using images of Brownian motion, stable Lévy process and fractional Brownian motion. Bluhm [8] has constructed Salem sets using statistically self-similar fractals. The results of Kahane [12], [13] and Bluhm [8] also determine the rate of $\widehat{\nu}(\xi) \rightarrow 0$ as $\xi \rightarrow \infty$, where $\nu$ is either the occupation measure of a stable Lévy process and fractional Brownian motion, or a statistically self-similar measure.

Deterministic Salem sets with non-integer Hausdorff dimension are more difficult to construct, there are only a few examples. Kaufman [18] has shown that for $a>0$, the set $E_{a}=\left\{x \in[0,1]: d(n x, \mathbb{N}) \leq n^{-(1+a)}\right.$ has solutions for arbitrarily large integers $n\}$ is a Salem set with Hausdorff dimension $\operatorname{dim}_{\mathrm{H}} E_{a}=2 /(2+a)$, where $d(x, \mathbb{N})$ denotes the distance of $x$ to the nearest integer. By modifying the method of Kaufman [18], Bluhm [6], [7] has provided an explicit Cantor-type construction of deterministic linear Salem sets with prescribed dimension. 
In this section, we continue the line of research of Kahane [12]-[14] and study the asymptotic properties of the Fourier transforms of the image measures of a large class of $(N, d)$-Gaussian random fields. In particular, we show that for every Borel set $E \subset \mathbb{R}^{N}$, under certain mild conditions, $X(E)$ is almost surely a Salem set.

First let $Y=\left\{Y(t), t \in \mathbb{R}^{N}\right\}$ be a real-valued, centered Gaussian random field with $Y(0)=0$. We assume that $Y$ has stationary increments and continuous covariance function $R(s, t)=\mathbb{E}[X(s) X(t)]$. According to Yaglom [33], $R(s, t)$ can be represented as

$$
R(s, t)=\int_{\mathbb{R}^{N}}\left(e^{i\langle s, \lambda\rangle}-1\right)\left(e^{-i\langle t, \lambda\rangle}-1\right) \Delta(d \lambda)+\langle s, Q t\rangle,
$$

where $Q$ is an $N \times N$ non-negative definite matrix and $\Delta(d \lambda)$ is a nonnegative symmetric measure on $\mathbb{R}^{N} \backslash\{0\}$ satisfying

$$
\int_{\mathbb{R}^{N}} \frac{|\lambda|^{2}}{1+|\lambda|^{2}} \Delta(d \lambda)<\infty .
$$

The measure $\Delta$ is called the spectral measure of $Y$.

It follows from (2.4) that $Y$ has the following stochastic integral representation:

$$
Y(t)=\int_{\mathbb{R}^{N}}\left(e^{i\langle t, \lambda\rangle}-1\right) W(d \lambda)+\langle\Xi, t\rangle,
$$

where $\Xi$ is an $N$-dimensional Gaussian random vector with mean 0 and $W(d \lambda)$ is a centered complex-valued Gaussian random measure which is independent of $\Xi$ and satisfies

$$
\mathbb{E}(W(A) \overline{W(B)})=\Delta(A \cap B) \text { and } W(-A)=\overline{W(A)}
$$

for all Borel sets $A, B \subseteq \mathbb{R}^{N}$. Throughout this paper, we will assume that $\Xi=0$. This is equivalent to assuming $Q=0$ in (2.4). Consequently, for all $h \in \mathbb{R}^{N}$ we have

$$
\sigma^{2}(h)=\mathbb{E}\left[(Y(t+h)-Y(t))^{2}\right]=2 \int_{\mathbb{R}^{N}}(1-\cos \langle h, \lambda\rangle) \Delta(d \lambda) .
$$

Besides $\sigma^{2}(h)$, we will make use of the function $\phi:[0, \infty) \rightarrow[0, \infty)$ defined by

$$
\phi(r)=\Delta\left\{\xi:|\xi| \geq r^{-1}\right\} \quad \text { for } r>0 \text { and } \phi(0)=0 .
$$

Then $\phi$ is a non-decreasing and right continuous on $[0, \infty)$. In this section, we will assume that the spectral measure $\Delta$ is absolutely continuous and its density function $f(\lambda)$ satisfies the following condition (when $N=1$, this is due to Berman [4]):

$$
0<\underline{\alpha}=\frac{1}{2} \liminf _{\lambda \rightarrow \infty} \frac{\vartheta_{N}|\lambda|^{N} f(\lambda)}{\phi(1 /|\lambda|)} \leq \frac{1}{2} \limsup _{\lambda \rightarrow \infty} \frac{\vartheta_{N}|\lambda|^{N} f(\lambda)}{\phi(1 /|\lambda|)}=\bar{\alpha}<1,
$$


where $\vartheta_{1}=2$ and for $N \geq 2, \vartheta_{N}=\mu\left(S^{N-1}\right)$ is the area (i.e., $(N-1)$ dimensional Lebesgue measure) of the unit sphere $S^{N-1}$ in $\mathbb{R}^{N}$.

Under the assumption that the spectral measure $\Delta$ has a density $f(\lambda)$, condition (2.8) is more general than assuming $\phi$ is regularly varying at 0 . In fact, by Theorem 2.1.1 in Bingham et al. [5] one can show that a necessary and sufficient condition for $\phi(r)$ to be regularly varying at 0 of index $2 \alpha$ is that the limit

$$
\alpha=\frac{1}{2} \lim _{r \rightarrow \infty} \frac{r^{N} \int_{S^{N-1}} f(r \theta) \mu(d \theta)}{\Delta\{\xi:|\xi| \geq r\}}
$$

exists; see Xiao [31] for more details.

The following Lemmas 2.1 and 2.2 are proved in Xiao [31]. They give some properties of $\phi$ and, more importantly, its connection with $\sigma^{2}(h)$ in (2.7).

Lemma 2.1. Assume (2.8) holds. Then for any $\varepsilon \in(0,2 \min \{\underline{\alpha}, 1-\bar{\alpha}\})$, there exists a constant $r_{0}>0$ such that for all $0<x \leq y \leq r_{0}$,

$$
\left(\frac{x}{y}\right)^{2 \bar{\alpha}+\varepsilon} \leq \frac{\phi(x)}{\phi(y)} \leq\left(\frac{x}{y}\right)^{2 \underline{\alpha}-\varepsilon} .
$$

In particular, the function $\phi$ has the following doubling property: there exists a constant $K_{2,1}>0$ such that for all $r>0$ small,

$$
\phi(2 r) \leq K_{2,1} \phi(r) .
$$

Using the terminology of Bingham et al. [5, pp. 65-67], (2.9) implies that $\phi$ is extended regularly varying at 0 with upper and lower Karamata indices $2 \bar{\alpha}$ and $2 \underline{\alpha}$, respectively.

LEMMA 2.2. Under the assumption (2.8), we have

$$
0<\liminf _{h \rightarrow 0} \frac{\sigma^{2}(h)}{\phi(|h|)} \leq \limsup _{h \rightarrow 0} \frac{\sigma^{2}(h)}{\phi(|h|)}<\infty .
$$

REMARK 2.3. In the studies of various properties of Gaussian random fields with stationary increments, the variance function $\sigma^{2}(h)$ has played a significant role and it is typically assumed to be regularly varying at 0 and/or monotone. See, for example, Kasahara et al. [16], Talagrand [25], [26], Xiao [28]-[30] and so on. With the help of Lemmas 2.1 and 2.2, Xiao [31] has shown that, under condition (2.8), it is more convenient to use $\phi(r)$ to investigate the sample path properties of Gaussian random fields and thus the regularly varying assumption on $\sigma^{2}(h)$ can be significantly weakened and the monotonicity assumption on $\sigma^{2}(h)$ can be removed.

The following are some examples of Gaussian random fields satisfying condition (2.8). 
EXAmple 2.4. Let $B_{\alpha}=\left\{B_{\alpha}(t), t \in \mathbb{R}^{N}\right\}$ be an $N$-parameter fractional Brownian motion of Hurst index $\alpha \in(0,1)$. Then its spectral density is given by

$$
f_{\alpha}(\lambda)=c(\alpha, N) \frac{1}{|\lambda|^{2 \alpha+N}},
$$

where $c(\alpha, N)>0$ is a normalizing constant. Clearly, $\underline{\alpha}=\bar{\alpha}=\alpha$ and $\sigma^{2}(h)=|h|^{2 \alpha}$.

EXAmple 2.5. Consider the mean zero Gaussian random field $Y=$ $\left\{Y(t), t \in \mathbb{R}^{N}\right\}$ in $\mathbb{R}$ with stationary increments and spectral density

$$
f_{\gamma, \beta}(\lambda)=\frac{c(\gamma, \beta, N)}{|\lambda|^{2 \gamma}\left(1+|\lambda|^{2}\right)^{\beta}},
$$

where $\beta$ and $\gamma$ are constants satisfying

$$
\beta+\gamma>N / 2, \quad 0<\gamma<1+N / 2,
$$

and $c(\gamma, \beta, N)>0$ is a normalizing constant. Since the spectral density $f_{\gamma, \beta}$ involves both the Fourier transforms of the Riesz kernel and the Bessel kernel, Anh et al. [2] call the corresponding Gaussian process the fractional Riesz-Bessel motion with indices $\beta$ and $\gamma$, and they have shown that these Gaussian random fields can be used for simultaneously modeling long range dependence and intermittency.

It is easy to check that for the spectral measure with density (2.12) the limit in condition (2.8) exists, i.e., $\underline{\alpha}=\bar{\alpha}=\gamma+\beta-N / 2$. Moreover, since the spectral density $f_{\gamma, \beta}(x)$ is regularly varying at infinity of order $2(\beta+\gamma)>N$, by a result of Pitman [23] we know that, if $\gamma+\beta-N / 2<1$, then $\sigma(h)$ is regularly varying at 0 of order $\beta+\gamma-N / 2$ and

$$
\sigma(h) \sim|h|^{\beta+\gamma-N / 2},
$$

where $a(h) \sim b(h)$ means $a(h) / b(h) \rightarrow 1$ as $h \rightarrow 0$. We will see that the results of this paper are applicable to the fractional Riesz-Bessel motion. For more examples of Gaussian random fields satisfying (2.8), see Xiao [31].

We also need the following lemma, which is a special case of Lemma 2.2 in Xiao [31].

Lemma 2.6. Assume that the spectral density $f$ satisfies condition (2.8). Then for any fixed constants $T>0$ and $K_{2,2}>0$, there exists a positive and finite constant $K_{2,3}$ such that for all functions $g$ of the form

$$
g(\lambda)=\sum_{j=1}^{n}\left(e^{i\left\langle t_{j}, \lambda\right\rangle}-e^{i\left\langle s_{j}, \lambda\right\rangle}\right) \quad \forall s_{j}, t_{j} \in[-T, T]^{N},
$$


we have

$$
|g(\lambda)| \leq K_{2,3}|\lambda|\left(\int_{\mathbb{R}^{N}}|g(\xi)|^{2} f(\xi) d \xi\right)^{1 / 2} \quad \text { for all }|\lambda|<K_{2,2} .
$$

Let $T>0$ be fixed. For all $n \geq 2, t_{1}, \ldots, t_{n}, s_{1}, \ldots, s_{n} \in[-T, T]^{N}$, write $\mathbf{s}=\left(s_{1}, \ldots, s_{n}\right), \mathbf{t}=\left(t_{1}, \ldots, t_{n}\right)$ and define

$$
\Psi(\mathbf{s}, \mathbf{t})=\mathbb{E}\left[\sum_{j=1}^{n}\left(Y\left(t_{j}\right)-Y\left(s_{j}\right)\right)\right]^{2}
$$

For $\mathbf{s} \in[-T, T]^{n N}$ and $r>0$, let

$$
F(\mathbf{s}, r)=\bigcup_{j=1}^{n} B\left(s_{j}, r\right)
$$

and

$$
G(\mathbf{s}, r)=\left\{\mathbf{t}=\left(t_{1}, \ldots, t_{n}\right): t_{k} \in F(\mathbf{s}, r) \text { for } 1 \leq k \leq n\right\} .
$$

The following lemma is essential for the proof of Theorem 2.8.

Lemma 2.7. Assume that the spectral measure $\Delta$ of $Y=\left\{Y(t), t \in \mathbb{R}^{N}\right\}$ has a density function $f$ that satisfies (2.8). Then there exists a constant $0<K_{2,4}<\infty$, depending on $\bar{\alpha}, \underline{\alpha}$ and $T$ only, such that for all $r \in\left(0, r_{0}\right)$ (with $r_{0}$ given in Lemma 2.1) and all $\mathbf{s}, \mathbf{t} \in[-T, T]^{n N}$ with $\mathbf{t} \notin G(\mathbf{s}, r)$, we have $\Psi(\mathbf{s}, \mathbf{t}) \geq K_{2,4} \phi(r)$.

Proof. By (2.8), we see that for every constant $\varepsilon \in(0,2 \min \{\underline{\alpha}, 1-\bar{\alpha}\})$, there exists a constant $r_{1} \in\left(0, r_{0} \wedge 1\right)$ such that

$$
2 \underline{\alpha}-\varepsilon<\frac{\vartheta_{N}|\lambda|^{N} f(\lambda)}{\phi(1 /|\lambda|)} \leq 2 \bar{\alpha}+\varepsilon \quad \text { for all } \lambda \in \mathbb{R}^{N} \text { with }|\lambda| \geq 1 / r_{1} \text {. }
$$

It follows from (2.4) that

$$
\Psi(\mathbf{t}, \mathbf{s})=\int_{\mathbb{R}^{N}}\left|\sum_{j=1}^{n}\left(e^{i\left\langle t_{j}, \lambda\right\rangle}-e^{i\left\langle s_{j}, \lambda\right\rangle}\right)\right|^{2} f(\lambda) d \lambda .
$$

Now we choose a bump function $\delta(\cdot) \in C^{\infty}\left(\mathbb{R}^{N}\right)$ with values in $[0,1]$ such that $\delta(0)=1$ and it vanishes outside the open unit ball. Let $\widehat{\delta}$ be the Fourier transform of $\delta$. It is known that $\widehat{\delta}(\lambda)$ is bounded and decays rapidly as $\lambda \rightarrow \infty$. Let $\delta_{r}(t)=r^{-N} \delta(t / r)$. Then the Fourier inversion formula gives

$$
\delta_{r}(t)=(2 \pi)^{-N} \int_{\mathbb{R}^{N}} e^{-i\langle t, \lambda\rangle} \widehat{\delta}(r \lambda) d \lambda .
$$

Note that since $\mathbf{t} \notin G(\mathbf{s}, r)$, there exists $t_{k}$ such that $\min \left\{\left|t_{k}-s_{j}\right|: 1 \leq j\right.$ $\leq n\} \geq r$. Hence $\delta_{r}\left(s_{j}-t_{k}\right)=0$ for all $j=1, \ldots, n$. It follows from $(2.19)$ that 


$$
\begin{aligned}
\int_{\mathbb{R}^{N}}\left(\sum_{j=1}^{n}\left(e^{i\left\langle t_{j}, \lambda\right\rangle}-e^{i\left\langle s_{j}, \lambda\right\rangle}\right)\right) e^{-i\left\langle t_{k}, \lambda\right\rangle} \widehat{\delta}(r \lambda) d \lambda \\
=(2 \pi)^{N} \sum_{j=1}^{n}\left(\delta_{r}\left(t_{j}-t_{k}\right)-\delta_{r}\left(s_{j}-t_{k}\right)\right) \geq(2 \pi)^{N} r^{-N}
\end{aligned}
$$

Next, we split the integral in (2.20) over $\left\{\lambda:|\lambda|<1 / r_{1}\right\}$ and $\{\lambda:|\lambda| \geq$ $\left.1 / r_{1}\right\}$. Denote the two integrals by $I_{1}$ and $I_{2}$, respectively. It follows from Lemma 2.6 with $K_{2,2}=1 / r_{1}$ that

$$
\begin{aligned}
I_{1} \leq & \int_{|\lambda|<1 / r_{1}}\left|\sum_{j=1}^{n}\left(e^{i\left\langle t_{j}, \lambda\right\rangle}-e^{i\left\langle s_{j}, \lambda\right\rangle}\right)\right||\widehat{\delta}(r \lambda)| d \lambda \\
\leq & K_{2,3}\left(\int_{\mathbb{R}^{N}}\left|\sum_{j=1}^{n}\left(e^{i\left\langle t_{j}, \xi\right\rangle}-e^{i\left\langle s_{j}, \xi\right\rangle}\right)\right|^{2} f(\xi) d \xi\right)^{1 / 2} \\
& \times \int_{|\lambda|<1 / r_{1}}|\lambda||\widehat{\delta}(r \lambda)| d \lambda \\
\leq & K_{2,4}[\Psi(\mathbf{s}, \mathbf{t})]^{1 / 2}
\end{aligned}
$$

where the last inequality follows from $(2.18)$ and the boundedness of $\widehat{\delta}$. On the other hand, by the Cauchy-Schwarz inequality and (2.18), we have

$$
\begin{aligned}
I_{2}^{2} \leq & \int_{|\lambda| \geq 1 / r_{1}}\left|\sum_{j=1}^{n}\left(e^{i\left\langle t_{j}, \lambda\right\rangle}-e^{i\left\langle s_{j}, \lambda\right\rangle}\right)\right|^{2} f(\lambda) d \lambda \\
& \times \int_{|\lambda| \geq 1 / r_{1}} \frac{1}{f(\lambda)}|\widehat{\delta}(r \lambda)|^{2} d \lambda \\
\leq & \Psi(\mathbf{s}, \mathbf{t}) \cdot r^{-N} \int_{|\lambda| \geq r / r_{1}} \frac{1}{f(\lambda / r)}|\widehat{\delta}(\lambda)|^{2} d \lambda .
\end{aligned}
$$

Note that by using (2.17) and Lemma 2.1, we deduce

$$
\begin{aligned}
& \int_{|\lambda| \geq r / r_{1}} \frac{1}{f(\lambda / r)}|\widehat{\delta}(\lambda)|^{2} d \lambda \\
& \quad \leq \vartheta_{N}(2 \underline{\alpha}-\varepsilon)^{-1} r^{-N} \int_{|\lambda| \geq r / r_{1}} \frac{|\lambda|^{N}}{\phi(r /|\lambda|)}|\widehat{\delta}(\lambda)|^{2} d \lambda \\
& \quad=\vartheta_{N}(2 \underline{\alpha}-\varepsilon)^{-1} \phi(r)^{-1} r^{-N} \int_{\substack{|\lambda| \geq r / r_{1}\\
}} \frac{\phi(r)}{\phi(r /|\lambda|)}|\lambda|^{N}|\widehat{\delta}(\lambda)|^{2} d \lambda \\
& \quad \leq K \phi(r)^{-1} r^{-N} \int_{\mathbb{R}^{N}}|\lambda|^{N+2 \bar{\alpha}+\varepsilon|\widehat{\delta}(\lambda)|^{2} d \lambda=K \phi(r)^{-1} r^{-N} .}
\end{aligned}
$$

Squaring both sides of (2.20) and combining (2.21), (2.22) and (2.23), we obtain 


$$
(2 \pi)^{2 N} r^{-2 N} \leq K \phi(r)^{-1} r^{-2 N} \Psi(\mathbf{s}, \mathbf{t}) .
$$

This gives the desired inequality.

Now we consider the $(N, d)$-Gaussian random field $X=\left\{X(t), t \in \mathbb{R}^{N}\right\}$ defined by

$$
X(t)=\left(X_{1}(t), \ldots, X_{d}(t)\right) \quad \forall t \in \mathbb{R}^{N},
$$

where $X_{1}, \ldots, X_{d}$ are independent copies of a real-valued, centered Gaussian random field $Y=\left\{Y(t), t \in \mathbb{R}^{N}\right\}$ with stationary increments and $Y(0)=0$. We assume that $Y$ has a spectral density $f$ that satisfies condition (2.8). Then Lemma 2.2 implies that for some positive constants $K_{2,5}$ and $K_{2,6}$,

$$
K_{2,5} \phi(|h|) \leq \mathbb{E}\left[(Y(t+h)-Y(t))^{2}\right] \leq K_{2,6} \phi(|h|)
$$

for all $t, h \in \mathbb{R}^{N}$ with $|h|$ small enough.

For any Borel probability measure $\mu$ on $\mathbb{R}^{N}$, let $\nu=\mu \circ X^{-1}$ be the image measure of $\mu$ under $X$. Then the Fourier transform of $\nu$ can be written as

$$
\widehat{\nu}(\xi)=\int_{\mathbb{R}^{N}} e^{i\langle\xi, X(t)\rangle} \mu(d t) .
$$

The following theorem describes the asymptotic behavior of $\widehat{\nu}(\xi)$ as $\xi \rightarrow \infty$.

TheOREM 2.8. Let $X=\left\{X(t), t \in \mathbb{R}^{N}\right\}$ be an $(N, d)$-Gaussian random field with stationary increments defined above, and let $\tau: \mathbb{R}_{+} \rightarrow \mathbb{R}_{+}$be a non-decreasing function satisfying the doubling property, that is, $\tau(2 r) \leq$ $K_{2,7} \tau(r)$ for all $r \geq 0$. If $\mu$ is a probability measure on $[-T, T]^{N}$ such that

$$
\mu(B(x, r)) \leq K_{2,8} \tau(2 r) \quad \forall x \in \mathbb{R}^{N}, r \geq 0,
$$

then there exists a positive and finite constant $\eta$ such that almost surely,

$$
\limsup _{|\xi| \rightarrow \infty} \frac{|\widehat{\nu}(\xi)|}{\sqrt{\tau\left(\phi^{-1}\left(|\xi|^{-2}\right)\right) \log ^{\eta}|\xi|}}<\infty
$$

where $\phi^{-1}(x)=\inf \{y: \phi(y)>x\}$ is the inverse function of $\phi$.

Proof. By considering the restriction of $\mu$ to subsets of its support and the linearity of the Fourier transform, we see that, without loss of generality, we may (and do) assume $\mu$ is supported on a Borel set $E \subset \mathbb{R}^{N}$ with $\operatorname{diam} E<r_{1}$. Hence Lemmas 2.1 and 2.7 are applicable.

For any positive integer $n \geq 1,(2.26)$ yields

$$
\begin{aligned}
\mathbb{E}\left(|\widehat{\nu}(\xi)|^{2 n}\right) & =\mathbb{E} \int_{\mathbb{R}^{2 n N}} e^{i\left\langle\xi, \sum_{j=1}^{n}\left(X\left(t_{j}\right)-X\left(s_{j}\right)\right)\right\rangle} \mu^{n}(d \mathbf{s}) \mu^{n}(d \mathbf{t}) \\
& =\int_{\mathbb{R}^{2 n N}} \exp \left(-\frac{1}{2}|\xi|^{2} \Psi(\mathbf{s}, \mathbf{t})\right) \mu^{n}(d \mathbf{s}) \mu^{n}(d \mathbf{t}),
\end{aligned}
$$

where $\mu^{n}(d \mathbf{s})=\mu\left(d s_{1}\right) \cdots \mu\left(d s_{n}\right)$. 
First we estimate the integral in (2.29) by integrating over $\mu^{n}(d \mathbf{t})$. Let $\mathbf{s}$ be fixed and write

$$
\begin{aligned}
\int_{\mathbb{R}^{n N}} \exp \left(-\frac{1}{2}|\xi|^{2} \Psi(\mathbf{s}, \mathbf{t})\right) \mu^{n}(d \mathbf{t}) \\
=\int_{G(\mathbf{s}, r)} \exp \left(-\frac{1}{2}|\xi|^{2} \Psi(\mathbf{s}, \mathbf{t})\right) \mu^{n}(d \mathbf{t}) \\
\quad+\sum_{k=1}^{\infty} \int_{G\left(\mathbf{s}, r 2^{k}\right) \backslash G\left(\mathbf{s}, r 2^{k-1}\right)} \exp \left(-\frac{1}{2}|\xi|^{2} \Psi(\mathbf{s}, \mathbf{t})\right) \mu^{n}(d \mathbf{t}) .
\end{aligned}
$$

By (2.27), we always have

$$
\int_{G(\mathbf{s}, r)} \exp \left(-\frac{1}{2}|\xi|^{2} \Psi(\mathbf{s}, \mathbf{t})\right) \mu^{n}(d \mathbf{t}) \leq\left(K_{2,8} n \tau(2 r)\right)^{n} .
$$

Given $\xi \in \mathbb{R}^{d} \backslash\{0\}$, we take $r=\phi^{-1}\left(|\xi|^{-2}\right)$. It follows from Lemma 2.7 and from (2.27) that

$$
\begin{aligned}
\int_{G\left(\mathbf{s}, r 2^{k}\right) \backslash G\left(\mathbf{s}, r 2^{k-1}\right)} & \exp \left(-\frac{1}{2}|\xi|^{2} \Psi(\mathbf{s}, \mathbf{t})\right) \mu^{n}(d \mathbf{t}) \\
\leq & \exp \left(-\frac{1}{2} K_{2,4}|\xi|^{2} \phi\left(r 2^{k-1}\right)\right) \cdot\left(K_{2,8} n \tau\left(2^{k+1} r\right)\right)^{n} \\
\leq & \left(K_{2,8} n \tau(2 r)\right)^{n} \exp \left(-K_{2,9} 2^{k(2 \underline{\alpha}-\varepsilon)}\right) \cdot K_{2,7}^{k n} .
\end{aligned}
$$

In deriving the last inequality, we have applied Lemma 2.1, the fact that $\phi\left(\phi^{-1}(x)\right) \geq x$ and the doubling property of the function $\tau$.

Combining (2.30)-(2.32), we derive an upper bound for the integral in (2.30):

$$
\begin{aligned}
\int_{\mathbb{R}^{n N}} \exp ( & \left.-\frac{1}{2}|\xi|^{2} \Psi(\mathbf{s}, \mathbf{t})\right) \mu^{n}(d \mathbf{t}) \\
& \leq\left(K_{2,8} n \tau(2 r)\right)^{n}\left(1+\sum_{k=1}^{\infty} \exp \left(-K_{2,9} 2^{k(2 \underline{\alpha}-\varepsilon)}\right) \cdot K_{2,7}^{k n}\right) \\
& \leq K_{2,10}^{n} n^{\eta n} \tau\left(2 \phi^{-1}\left(|\xi|^{-2}\right)\right)^{n},
\end{aligned}
$$

where $\eta=1+\log K_{2,7} /(2 \underline{\alpha}-\varepsilon)$. Integrating both sides of $(2.33)$ over $\mu^{n}(d \mathbf{s})$ and recalling (2.29), we obtain the following moment estimate:

$$
\mathbb{E}\left(|\widehat{\nu}(\xi)|^{2 n}\right) \leq K_{2,10}^{n} n^{\eta n} \tau\left(2 \phi^{-1}\left(|\xi|^{-2}\right)\right)^{n}
$$

for all integers $n \geq 1$.

Using the same argument as in Kahane [12, pp. 254-255], we deduce that (2.34) implies that almost surely

$$
\limsup _{m \in \mathbb{Z}^{d},|m| \rightarrow \infty} \frac{|\widehat{\nu}(m)|}{\sqrt{\tau\left(2 \phi^{-1}\left(|m|^{-2}\right)\right)(\log |m|)^{\eta}}}<\infty .
$$


Therefore (2.28) follows from (2.35) and [12, Lemma 1, p. 252]. This finishes the proof of Theorem 2.8.

REMARK 2.9. In Kahane [12], there seems to be an error in the proof on p. 266 between lines -7 and -5 . Inequality (9) there should be modified as in (2.34). Consequently, the conclusion of his Theorem 1 on page 267 should be revised as in (2.28).

For a given Borel set $E \subset \mathbb{R}^{N}$, Theorem 2.8 allows us to determine easily the Fourier dimension of $X(E)$ for an $(N, d)$-Gaussian random field $X$ satisfying (2.8).

COROLlARY 2.10. In addition to the hypotheses of Theorem 2.8, assume that for some positive constants $0<\alpha<1, K_{2,11}$ and $K_{2,12}$,

$$
K_{2,11} r^{2 \alpha} \leq \phi(r) \leq K_{2,12} r^{2 \alpha} \quad \forall r>0 \text { small enough }
$$

Then, for every Borel set $E \subset \mathbb{R}^{N}$ with $\operatorname{dim}_{\mathrm{H}} E \leq \alpha d, X(E)$ is almost surely a Salem set with Fourier dimension $\alpha^{-1} \operatorname{dim}_{\mathrm{H}} E$.

REMARK 2.11. Condition (2.36) is satisfied if the spectral density $f(\lambda)$ of $Y$ satisfies

$$
\begin{aligned}
K_{2,13}|\lambda|^{-(2 \alpha+N)} \leq f(\lambda) & \leq K_{2,14}|\lambda|^{-(2 \alpha+N)} \\
& \forall \lambda \in \mathbb{R}^{N} \text { with }|\lambda| \text { large enough, }
\end{aligned}
$$

where $0<\alpha<1, K_{2,13}$ and $K_{2,14}$ are positive and finite constants. In order for condition (2.8) to hold, we require $K_{2,14} \alpha / K_{2,13}<1$. Clearly, this condition is satisfied by both fractional Brownian motion $B_{\alpha}$ and fractional Riesz-Bessel motion with indices $\beta$ and $\gamma$ such that $\gamma+\beta-N / 2<1$.

Proof of Corollary 2.10. Without loss of generality, we assume $E$ is a bounded set, thus $E \subseteq[-T, T]^{N}$ for some constant $T>0$. For any $\gamma \in$ $\left(0, \operatorname{dim}_{\mathrm{H}} E\right)$, Frostman's lemma implies that there is a probability measure $\mu$ on $E$ such that $\mu(B(x, r)) \leq K r^{\gamma}$ for all $x \in \mathbb{R}^{N}$ and $r>0$. Again, let $\nu$ be the image measure of $\mu$ under $X$. Then Theorem 2.8 and (2.36) imply that almost surely for some $K=K(\omega)$,

$$
|\widehat{\nu}(\xi)| \leq K|\xi|^{-\gamma /(2 \alpha)}(\log |\xi|)^{\eta / 2} \quad \forall \xi \in \mathbb{R}^{d} \backslash\{0\} .
$$

Thus $\operatorname{dim}_{\mathrm{F}} X(E) \geq \gamma / \alpha$ almost surely. Since $\gamma \in\left(0, \operatorname{dim}_{\mathrm{H}} E\right)$ is arbitrary, this yields $\operatorname{dim}_{\mathrm{F}} X(E) \geq \alpha^{-1} \operatorname{dim}_{\mathrm{H}} E$ a.s.

On the other hand, (2.36) and Lemma 2.2 imply that $X(t)$ satisfies almost surely a uniform Hölder condition on $[-T, T]^{N}$ of all orders smaller than $\alpha$. Hence we have $\operatorname{dim}_{\mathrm{H}} X(E) \leq \alpha^{-1} \operatorname{dim}_{\mathrm{H}} E$ a.s. (cf. Kahane [12, Chapter 10]). It follows that $\operatorname{dim}_{\mathrm{F}} X(E)=\operatorname{dim}_{\mathrm{H}} X(E)=\alpha^{-1} \operatorname{dim}_{\mathrm{H}} E$ almost surely. Therefore, $X(E)$ is almost surely a Salem set.

By examining the proof of Theorem 2.8, we see that (2.28) holds as long as $\Psi(\mathbf{s}, \mathbf{t}) \geq K \phi(r)$ if $\mathbf{t} \notin G(\mathbf{s}, r)$. Hence, we can provide a more general 
result on Salem sets for $(N, d)$-Gaussian random fields; see Theorem 2.12 below.

For this purpose, we only assume $Y=\left\{Y(t), t \in \mathbb{R}^{N}\right\}$ is a real-valued, centered Gaussian random field satisfying the following condition $(\mathrm{C} 1)$ :

(i) There exist positive constants $\delta_{0}, K_{2,15} \leq K_{2,16}$ and a non-decreasing, continuous function $\psi:\left[0, \delta_{0}\right) \rightarrow[0, \infty)$ such that for all $t \in \mathbb{R}^{N}$ and $h \in \mathbb{R}^{N}$ with $|h| \leq \delta_{0}$,

$$
K_{2,15} \psi(|h|) \leq \mathbb{E}\left[(Y(t+h)-Y(t))^{2}\right] \leq K_{2,16} \psi(|h|) .
$$

(ii) Let $\Psi(\mathbf{s}, \mathbf{t})$ and $G(\mathbf{s}, r)$ be defined by (2.15) and (2.16), respectively. Then there exists a constant $K_{2,17}>0$ such that

$$
\Psi(\mathbf{s}, \mathbf{t}) \geq K_{2,17} \psi(r) \quad \text { if } \mathbf{t} \notin G(\mathbf{s}, r) .
$$

Theorem 2.12. Let $X=\left\{X(t), t \in \mathbb{R}^{N}\right\}$ be an $(N, d)$-Gaussian random field defined by (2.24) with $Y$ satisfying condition (C1). Moreover, assume that the function $\psi$ is of order $2 \alpha$, that is,

$$
2 \alpha=\inf \left\{\beta \geq 0: \lim _{r \rightarrow 0} \frac{\psi(r)}{|r|^{\beta}}=\infty\right\}=\sup \left\{\beta \geq 0: \lim _{r \rightarrow 0} \frac{\psi(r)}{|r|^{\beta}}=0\right\},
$$

and $\alpha \in(0,1)$. Then for every Borel set $E \subset \mathbb{R}^{N}$ with $\operatorname{dim}_{\mathrm{H}} E \leq \alpha d, X(E)$ is almost surely a Salem set with Fourier dimension $\alpha^{-1} \operatorname{dim}_{\mathrm{H}} E$.

Proof. As mentioned above, the proof of Theorem 2.8 shows that the condition (2.39) implies (2.28) (with $\phi$ replaced by $\psi$ ) for every $\mu$ satisfying (2.27). Hence the proof of Theorem 2.12 is similar to that of Corollary 2.10 with obvious modifications and so the details are omitted.

We expect that Theorem 2.12 and Theorem 3.2 in Section 3 are applicable to a wide class of Gaussian random fields with stationary increments and discrete spectral measures. A systematic treatment for such Gaussian random fields will have to be done elsewhere because this would require methods that are different from those in Xiao [31] and the present paper. In the following, we only give an example of a stationary Gaussian process with discrete spectrum that satisfies condition $(\mathrm{C} 1)$, as well as condition $(\mathrm{C} 2)$ of Section 3.

EXAMPLE 2.13. Let $\left\{X_{n}, Y_{n}, n \geq 0\right\}$ be a sequence of independent standard normal random variables. Then for each $t \in \mathbb{R}$, the random Fourier series

$$
Y(t)=\frac{\sqrt{8}}{\pi} \sum_{n=0}^{\infty} \frac{1}{2 n-1}\left(X_{n} \cos ((2 n-1) t)+Y_{n} \sin ((2 n-1) t)\right)
$$

converges almost surely (see Kahane [12]), and $Y=\{Y(t), t \in \mathbb{R}\}$ is a centered, periodic and stationary Gaussian process with mean 0 and covariance function 


$$
R(s, t)=1-\frac{2}{\pi}|s-t| \quad \text { for }-\pi \leq s-t \leq \pi .
$$

It can be verified that the spectrum measure $\Delta$ of $Y$ is discrete and satisfies $\Delta(\{2 n-1\})=(2 n-1)^{-2}$ for all $n \in \mathbb{N}$, and that (2.38) holds with $\psi(r)=r$. Now we show that (2.39) also holds.

Lemma 2.14. Let $Y$ be the stationary Gaussian process defined by (2.41). Then for any interval $I \subset[-\pi, \pi]$ with length $|I| \leq \pi / 2$ there exists a constant $0<K_{2,18}<\infty$ such that

(i) for all $r \in(0, \pi / 2)$ and all $\mathbf{s}, \mathbf{t} \in I^{n}$ with $\mathbf{t} \notin G(\mathbf{s}, r)$, we have $\Psi(\mathbf{s}, \mathbf{t}) \geq K_{2,18} r$

(ii) for all $t \in I$ and all $0<r \leq \min \{|t|, \pi / 2\}$,

$$
\operatorname{Var}(Y(t)|Y(s): s \in I,| s-t \mid \geq r) \geq K_{2,18} r .
$$

Proof. Since $|I| \leq \pi / 2$ it is easy to verify that the covariance function $R(s, t)$ in (2.42) is biconvex on $I$ in the sense of Berman [3]. Write $I=$ $[a, b]$. It follows from Theorem 2.1 in [3] that $Y=\{Y(t), t \in I\}$ has the representation

$$
Y(t)=\xi_{1} \sqrt{R(a, b)}+\frac{2}{\pi}\left(B_{1}(t)-B_{1}(a)\right)+\frac{2}{\pi}\left(B_{2}(b)-B_{2}(t)\right),
$$

where $\xi_{1}$ is a standard normal random variable, $B_{1}=\{B(t), t \in I\}$ and $B_{2}=$ $\left\{B_{2}(t), t \in I\right\}$ are Brownian motions on $I$. All of the processes on the righthand side of (2.44) are mutually independent. Of course, the above representation can also be checked directly by verifying the covariance functions.

Since statement (i) of Lemma 2.7 holds for Brownian motion on $I$ (see Kahane [12, p. 254]), we derive from (2.44) that

$$
\Psi(\mathbf{s}, \mathbf{t}) \geq 2\left(\frac{2}{\pi}\right)^{2} \mathbb{E}\left[\sum_{j=1}^{n}\left(B_{1}\left(t_{j}\right)-B_{1}\left(s_{j}\right)\right)\right]^{2} \geq K_{2,18} r .
$$

To prove (2.43), it is sufficient to show that for all positive integers $k \geq 1$, all $s_{j} \in I$ with $\left|t-s_{j}\right| \geq r$ and $c_{j} \in \mathbb{R}(1 \leq j \leq k)$, we have

$$
\mathbb{E}\left[Y(t)-\sum_{j=1}^{k} c_{j} Y\left(s_{j}\right)\right]^{2} \geq K_{2,18} r .
$$

By using (2.44) again, we have

$$
\begin{aligned}
\mathbb{E}\left[Y(t)-\sum_{j=1}^{k} c_{j} Y\left(s_{j}\right)\right]^{2} \geq & \left(\frac{2}{\pi}\right)^{2} \mathbb{E}\left[B_{1}(t)-\sum_{j=0}^{k} c_{j} B_{1}\left(s_{j}\right)\right]^{2} \\
& +\left(\frac{2}{\pi}\right)^{2} \mathbb{E}\left[B_{2}(t)-\sum_{j=1}^{k+1} c_{j} B_{2}\left(s_{j}\right)\right]^{2}
\end{aligned}
$$


where $s_{0}=a, s_{k+1}=b$ and

$$
c_{0}=c_{k+1}=1-\sum_{j=1}^{k} c_{j} .
$$

Hence (2.46) follows from (2.47) and the property of independent increments of Brownian motion; see Lemma 4.3 in Khoshnevisan and Xiao [21] for the details. This finishes the proof of Lemma 2.14.

We end this section with the following remark. There are other natural random measures associated to a Gaussian random field $X$ such as the induced measure on the graph set $\operatorname{Gr} X(E)=\{(t, X(t)): t \in E\}$ and the local time measure on the level set $X^{-1}(x)=\left\{t \in \mathbb{R}^{N}: X(t)=x\right\}$. It is of interest to study the asymptotic properties of the Fourier transforms of these random measures at infinity, but they are more difficult to handle. The following is an open question of Kahane [14, p. 155].

QUESTION 2.15. Are the graph and level sets of a stochastic process such as fractional Brownian motion Salem sets?

3. Interior points. In this section, we consider the images of a Gaussian random field $X=\left\{X(t), t \in \mathbb{R}^{N}\right\}$ in $\mathbb{R}^{d}$ defined by

$$
X(t)=\left(X_{1}(t), \ldots, X_{d}(t)\right) \quad \forall t \in \mathbb{R}^{N},
$$

where $X_{1}, \ldots, X_{d}$ are independent copies of a real-valued, centered Gaussian random field $Y=\left\{Y(t), t \in \mathbb{R}^{N}\right\}$. We assume the following condition (C2):

(i) There exist positive constants $\delta_{1}, K_{3,1} \leq K_{3,2}$ and a non-decreasing, continuous function $\psi:\left[0, \delta_{1}\right) \rightarrow[0, \infty)$ such that for all $t \in \mathbb{R}^{N}$ and $h \in \mathbb{R}^{N}$ with $|h| \leq \delta_{1}$,

$$
K_{3,1} \psi(|h|) \leq \mathbb{E}\left[(Y(t+h)-Y(t))^{2}\right] \leq K_{3,2} \psi(|h|) .
$$

(ii) For some interval $I=[a, b] \subseteq \mathbb{R}^{N}, Y$ is strongly locally $\psi$-nondeterministic on $I$, that is, there exist positive constants $K_{3,3}$ and $r_{2}$ such that for all $t \in I$ and all $0<r \leq \min \left\{|t|, r_{2}\right\}$,

$$
\operatorname{Var}\left(Y(t)|Y(s): s \in I, r \leq| s-t \mid \leq r_{2}\right) \geq K_{3,3} \psi(r) .
$$

Remark 3.1. Condition (C2) is very useful in studying sample path properties of Gaussian random fields; see Xiao [31] and the references therein. It can be seen that conditions $(\mathrm{C} 2)$ and $(\mathrm{C} 1)$ are closely related. In particular, if a centered Gaussian random field $Y=\left\{Y(t), t \in \mathbb{R}^{N}\right\}$ has stationary increments with its spectral density $f(\lambda)$ satisfying (2.8), then Lemmas 2.1, 2.2 and 2.7 in Section 2 and Theorem 2.5 in Xiao [31] imply that both conditions $(\mathrm{C} 1)$ and $(\mathrm{C} 2)$ are satisfied with $\psi(r)=\phi(r)$. Example 2.13 shows that this may still be true even if the spectral measure of $Y$ is not absolutely 
continuous. More examples of Gaussian random fields satisfying both conditions $(\mathrm{C} 1)$ and $(\mathrm{C} 2)$ can be found in Xiao [31]. It is of interest to further study the connections between these two conditions.

Using the Fourier transforms, it is easy to prove (cf. Kahane [12]) that, under (i) of condition (C2), if a Borel set $E \subset \mathbb{R}^{N}$ carries a probability measure $\mu$ such that

$$
\iint_{E} \frac{1}{\psi(|s-t|)^{d / 2}} \mu(d s) \mu(d t)<\infty,
$$

then almost surely, $X(E)$ has positive $d$-dimensional Lebesgue measure. It is a natural question to further study when $X(E)$ has interior points. This question for Brownian motion was first considered by Kaufman [17], and then extended by Pitt [24] and Kahane [12], [13] to fractional Brownian motion.

The following is our main result of this section, which gives a sufficient condition for $X(E)$ to have interior points. When applied to fractional Brownian motion, Theorem 3.2 improves the results of Kaufman [17], Pitt [24] and Kahane [12], [13] mentioned in the introduction.

Theorem 3.2. Let $X=\left\{X(t), t \in \mathbb{R}^{N}\right\}$ be an $(N, d)$-Gaussian random field defined by (3.1) with $Y$ satisfying condition (C2). If a Borel set $E \subset I$ has positive capacity with respect to the kernel $\kappa(r)=\psi(r)^{-d / 2} \log ^{2 \gamma}(1 / \psi(r))$ with $\gamma>1$, that is, $E$ carries a probability measure $\mu$ such that

$$
\sup _{t \in \mathbb{R}^{N}} \int_{E} \frac{1}{\psi(|s-t|)^{d / 2}} \log ^{2 \gamma}\left(\frac{1}{\psi(|s-t|)}\right) \mu(d s) \leq K_{3,4}
$$

for some finite constant $K_{3,4}>0$, then $X(E)$ has interior points almost surely.

The following is a direct consequence of Theorem 3.2 and Frostman's theorem (cf. Kahane [12] or Khoshnevisan [20]). It is more convenient to use.

Corollary 3.3. Under the conditions of Theorem 3.2, let $\alpha^{*}$ be the upper index of $Y$ defined by

$$
\alpha^{*}=\frac{1}{2} \inf \left\{\beta \geq 0: \lim _{r \rightarrow 0} \frac{\psi(r)}{|r|^{\beta}}=\infty\right\} .
$$

If $E \subset I$ is a Borel set with $\operatorname{dim}_{\mathrm{H}} E>\alpha^{*} d$, then $X(E)$ a.s. has interior points.

For the proof of Theorem 3.2, we need several lemmas. Lemmas 3.4 and 3.6 are due to Cuzick and DuPreez [9], and Lemma 3.5 is a slight modification of their Lemma 3. 
Lemma 3.4. Let $\left\{Z_{i}\right\}_{i=1}^{n}$ be linearly independent centered Gaussian variables. If $g: \mathbb{R} \rightarrow \mathbb{R}_{+}$is a Borel measurable function, then

$$
\int_{\mathbb{R}^{n}} g\left(v_{1}\right) e^{-\operatorname{Var}(\langle v, Z\rangle) / 2} d v=\frac{(2 \pi)^{(n-1) / 2}}{Q^{1 / 2}} \int_{-\infty}^{\infty} g\left(z / \sigma_{1}\right) e^{-z^{2} / 2} d z,
$$

where $\sigma_{1}^{2}=\operatorname{Var}\left(Z_{1} \mid Z_{2}, \ldots, Z_{n}\right)$, and $Q=\operatorname{det} \operatorname{Cov}\left(Z_{1}, \ldots, Z_{n}\right)$ denotes the determinant of the covariance matrix of $Z$.

Lemma 3.5. Assume $p(y)$ is positive and non-decreasing on $(0, \infty)$, $p(0)=0, y^{n} / p^{n}(y)$ is non-decreasing on $[0,1]$, and $\int_{1}^{\infty} p^{-2}(y) d y<\infty$. Then there exists a constant $K_{3,5}$ such that for all integers $n \geq 1$,

$$
\int_{0}^{\infty} \frac{|\exp (i v y)-1|^{n}}{p^{n}(y)} d y \leq K_{3,5}^{n} p_{+}^{-n}(1 / v),
$$

where $p_{+}(y)=\min \{1, p(y)\}$.

Lemma 3.6. For $\alpha \geq e^{2} / 2$,

$$
\int_{1}^{\infty}(\log x)^{\alpha} \exp \left(-x^{2} / 2\right) d x \leq \sqrt{\pi}(\log \alpha)^{\alpha} .
$$

We will also make use of the following elementary formula to estimate the determinant of the covariance matrix of a Gaussian vector $Z$ :

$$
\operatorname{det} \operatorname{Cov}\left(Z_{1}, \ldots, Z_{n}\right)=\operatorname{Var}\left(Z_{1}\right) \prod_{j=2}^{n} \operatorname{Var}\left(Z_{j} \mid\left\{Z_{i}\right\}_{i \leq j-1}\right) .
$$

For completeness, we state the following basic result of Garsia [10].

Lemma 3.7 (Garsia's lemma). Assume that $p(u)$ and $\Psi(u)$ are two positive increasing functions on $[0, \infty), p(u) \downarrow 0$ as $u \downarrow 0, \Psi(u)$ is convex and $\Psi(u) \uparrow \infty$ as $u \uparrow \infty$. Let $D$ denote an open hypercube in $\mathbb{R}^{d}$. If the function $f(x)$ is measurable in $D$ and

$$
A:=A(D, f)=\iint_{D} \Psi\left(\frac{|f(x)-f(y)|}{p(|x-y| / \sqrt{d})}\right) d x d y<\infty,
$$

then after modifying $f(x)$ on a set of Lebesgue measure 0 , we have

$$
|f(x)-f(y)| \leq 8 \int_{0}^{|x-y|} \Psi^{-1}\left(A / u^{2 d}\right) d p(u) \quad \text { for all } x, y \in D .
$$

Proof of Theorem 3.2. By the Choquet capacity theorem, we may (and do) assume that $E$ is compact. Moreover, we assume that diam $E$ is small such that, say, $\psi(\operatorname{diam} E)<1 / e$.

As in Section 2, let $\nu$ be the image measure of $\mu$ under the mapping $t \mapsto X(t)$. If $\nu \ll \lambda_{d}$, where $\lambda_{d}$ is the Lebesgue measure in $\mathbb{R}^{d}$, then $X$ is 
said to have a local time on $E$. The local time $l_{\mu}(x)$ is defined to be the Radon-Nikodým derivative $d \nu / d \lambda_{d}(x)$ and it satisfies the following occupation density formula: for all Borel measurable functions $f: \mathbb{R}^{d} \rightarrow \mathbb{R}_{+}$,

$$
\int_{E} f(X(s)) \mu(d s)=\int_{\mathbb{R}^{d}} f(x) l_{\mu}(x) d x .
$$

It is known from Geman and Horowitz [11] or Kahane [12] that, when (3.4) holds, $l_{\mu}(x) \in L^{2}\left(\mathbb{R}^{d}\right)$ a.s.

Since $X(E)$ is a compact subset of $\mathbb{R}^{d},(3.12)$ implies that $\left\{x: l_{\mu}(x)>0\right\}$ is a subset of $X(E)$. Hence, in order to prove our theorem, it is sufficient to prove that the local time $l_{\mu}(x)$ has a version which is continuous in $x$; see Pitt [24, p. 324] or Geman and Horowitz [11, p. 12]. This will be proved by the moment method and Garsia's continuity lemma.

Let $\gamma>1$ be the constant in Theorem 3.2. We define

$$
p(u)= \begin{cases}0 & \text { if } u=0, \\ \log ^{-\gamma}(e \sqrt{d} /|u|) & \text { if } 0<|u| \leq 1, \\ \gamma|u|-\gamma+1 & \text { if }|u|>1\end{cases}
$$

Clearly, the function $p(u)$ is symmetric on $\mathbb{R}$, strictly increasing on $[0, \infty)$ and $p(u) \downarrow 0$ as $u \downarrow 0$.

The following lemma gives the key estimate for finishing the proof of Theorem 3.2.

Lemma 3.8. Let $D \subset \mathbb{R}^{d}$ be a hypercube. Then there exists a finite constant $K_{3,6}>0$, depending on $N, d, \gamma, \mu$ and $D$ only, such that for all even integers $n \geq 2$,

$$
\mathbb{E} \iint_{D}\left(\frac{l_{\mu}(x)-l_{\mu}(y)}{p(|x-y| / \sqrt{d})}\right)^{n} d x d y \leq K_{3,6}^{n} n !(\log n)^{n \gamma} .
$$

We now continue with the proof of Theorem 3.2 and defer the proof of Lemma 3.8 to the end of this section.

Let $\Psi(u)=u \exp \left(u^{\theta}\right)$, where $\theta \in(1 / \gamma, 1)$ is a constant. Then $\Psi$ is increasing and convex on $(0, \infty)$. It follows from Jensen's inequality and Lemma 3.8 that for all closed hypercubes $D \subset \mathbb{R}^{d}$ and all integers $n$ with $\theta+1 / n<1$,

$$
\begin{aligned}
& \mathbb{E} \int_{D}\left(\frac{\left|l_{\mu}(x)-l_{\mu}(y)\right|}{p(|x-y| / \sqrt{d})}\right)^{n \theta+1} d x d y \\
& \leq K\left\{\mathbb{E} \int_{D} \int_{D}\left(\frac{\left|l_{\mu}(x)-l_{\mu}(y)\right|}{p(|x-y| / \sqrt{d})}\right)^{n} d x d y\right\}^{\theta+1 / n} \\
& \leq K^{n}(n !)^{\theta+1 / n}(\log n)^{n \gamma(\theta+1 / n)} \leq K_{3,7}^{n}(n !)^{\theta}(\log n)^{n \gamma \theta},
\end{aligned}
$$

where $K_{3,7}$ is a finite constant depending on $N, d, \theta, D$ and $K_{3,6}$ only. 
Expanding $\Psi(u)$ into a power series and applying the above inequality, we derive

$$
\begin{aligned}
\mathbb{E} \iint_{D} \Psi\left(\frac{\left|l_{\mu}(x)-l_{\mu}(y)\right|}{p(|x-y| / \sqrt{d})}\right) d x d y \\
=\sum_{n=0}^{\infty} \frac{1}{n !} \mathbb{E} \iint_{D}\left(\frac{\left|l_{\mu}(x)-l_{\mu}(y)\right|}{p(|x-y| / \sqrt{d})}\right)^{n \theta+1} d x d y<\infty
\end{aligned}
$$

the last inequality follows from the fact that $\theta<1$. Hence Garsia's lemma implies that there are positive and finite random variables $A_{1}$ and $A_{2}$ such that for almost all $x, y \in D$ with $|x-y| \leq e^{-1}$,

$$
\left|l_{\mu}(x)-l_{\mu}(y)\right| \leq \int_{0}^{|x-y|} \Psi^{-1}\left(A_{1} / u^{2 d}\right) d p(u) \leq A_{2}[\log (1 /|x-y|)]^{-(\gamma-1 / \theta)} .
$$

Since we have chosen $\theta$ in such a way that $\gamma>1 / \theta$, we see that $X$ has almost surely a local time $l_{\mu}(x)$ on $E$ that is continuous for all $x \in D$. By taking an increasing sequence $\left\{D_{n}: n \geq 1\right\}$ of closed hypercubes such that $\mathbb{R}^{d}=\bigcup_{n=1}^{\infty} D_{n}$, we have proved that almost surely $l_{\mu}(x)$ is continuous for all $x \in \mathbb{R}^{\bar{d}}$. This completes the proof of Theorem 3.2.

It remains to prove Lemma 3.8.

Proof of Lemma 3.8. By equation (25.7) in Geman and Horowitz [11], we have, for every $x, y \in \mathbb{R}^{d}$ and all even integers $n \geq 2$,

$$
\begin{aligned}
\mathbb{E}\left[\left(l_{\mu}(x)-l_{\mu}(y)\right)^{n}\right]= & (2 \pi)^{-n d} \int_{E^{n}} \int_{\mathbb{R}^{n d}} \prod_{j=1}^{n}\left[e^{-i\left\langle u_{j}, x\right\rangle}-e^{-i\left\langle u_{j}, y\right\rangle}\right] \\
& \times \exp \left[-\frac{1}{2} \operatorname{Var}\left(\sum_{j=1}^{n}\left\langle u_{j}, X\left(t_{j}\right)\right\rangle\right)\right] d \mathbf{u} \mu^{n}(d \mathbf{t}) .
\end{aligned}
$$

In the above, $\mathbf{u}=\left(u_{1}, \ldots, u_{n}\right), u_{j} \in \mathbb{R}^{d}$ for each $j=1, \ldots, n$ and we will write it coordinatewise as $u_{j}=\left(u_{j}^{1}, \ldots, u_{j}^{d}\right)$.

Note that for $u_{1}, \ldots, u_{n}, y \in \mathbb{R}^{d}$, the triangle inequality implies

$$
\begin{aligned}
& \prod_{j=1}^{n}\left|\exp \left(-i\left\langle u_{j}, y\right\rangle\right)-1\right| \\
& \quad=\prod_{j=1}^{n}\left|\exp \left(-i \sum_{l=1}^{d} u_{j}^{l} y^{l}\right)-1\right| \\
& \leq \prod_{j=1}^{n}\left|\sum_{k=1}^{d}\left[\exp \left(-i \sum_{l=0}^{k} u_{j}^{l} y^{l}\right)-\exp \left(-i \sum_{l=0}^{k-1} u_{j}^{l} y^{l}\right)\right]\right|
\end{aligned}
$$




$$
\leq \prod_{j=1}^{n}\left[\sum_{k=1}^{d}\left|\exp \left(-i u_{j}^{k} y^{k}\right)-1\right|\right]=\sum^{\prime} \prod_{j=1}^{n}\left|\exp \left(-i u_{j}^{k_{j}} y^{k_{j}}\right)-1\right|,
$$

where $y^{0}=u_{j}^{0}=0$ and the summation $\sum^{\prime}$ is over all sequences $\left(k_{1}, \ldots, k_{n}\right)$ $\in\{1, \ldots, d\}^{n}$.

Hence for any fixed hypercube $D \subset \mathbb{R}^{d}$ and any even integer $n \geq 2$, we have

$$
\begin{aligned}
\mathbb{E} \int_{D} \int_{D}\left(\frac{l_{\mu}(x)-l_{\mu}(y)}{p(|x-y| / \sqrt{d})}\right)^{n} d x d y \\
\leq(2 \pi)^{-n d} \sum^{\prime} \iint_{D} \int_{E^{n}} \int_{\mathbb{R}^{n d}} \prod_{j=1}^{n} \frac{\left|\exp \left(i u_{j}^{k_{j}}\left(y^{k_{j}}-x^{k_{j}}\right)\right)-1\right|}{p(|y-x| / \sqrt{d})} \\
\quad \times \exp \left[-\frac{1}{2} \operatorname{Var}\left(\sum_{j=1}^{n}\left\langle u_{j}, X\left(t_{j}\right)\right\rangle\right)\right] d \mathbf{u} \mu^{n}(d \mathbf{t}) d x d y \\
\leq(2 \pi)^{-n d} \lambda_{d}(D) \sum^{\prime} \int_{D \ominus D} \int_{E^{n}} \prod_{\mathbb{R}^{n d}} \prod_{j=1}^{n} \frac{\left|\exp \left(i u_{j}^{k_{j}} y^{k_{j}}\right)-1\right|}{p\left(\left|y^{k_{j}}\right| / \sqrt{d}\right)} \\
\quad \times \exp \left[-\frac{1}{2} \operatorname{Var}\left(\sum_{j=1}^{n}\left\langle u_{j}, X\left(t_{j}\right)\right\rangle\right)\right] d \mathbf{u} \mu^{n}(d \mathbf{t}) d y .
\end{aligned}
$$

In the above, $D \ominus D=\{x-y: x, y \in D\}$. We have made a change of variables and have used the fact that $p(|y| / \sqrt{d}) \geq p\left(\left|y^{k}\right| / \sqrt{d}\right)$ for all $k=1, \ldots, d$. Also note that (3.5) implies that $\mu$ has no atom and thus Fubini's theorem implies that

$$
\mu^{n}\left\{\mathbf{t}=\left(t_{1}, \ldots, t_{n}\right): t_{i}=t_{j} \text { for some } i \neq j\right\}=0 .
$$

Hence the integral in (3.18) with respect to $\mu^{n}$ can be taken over the set $E_{\neq}^{n}=\left\{\mathbf{t} \in E^{n}: t_{1}, \ldots, t_{n}\right.$ are distinct $\}$.

Now we fix $\mathbf{t} \in E_{\neq}^{n}$, a sequence $\left(k_{1}, \ldots, k_{n}\right) \in\{1, \ldots, d\}^{n}$ and consider the integral

$$
\begin{aligned}
\mathcal{M}_{n}= & \int_{D \ominus D} \int_{\mathbb{R}^{n d}} \prod_{j=1}^{n} \frac{\left|\exp \left(i u_{j}^{k_{j}} y^{k_{j}}\right)-1\right|}{p\left(\left|y^{k_{j}}\right| / \sqrt{d}\right)} \\
& \times \exp \left[-\frac{1}{2} \operatorname{Var}\left(\sum_{j=1}^{n}\left\langle u_{j}, X\left(t_{j}\right)\right)\right] d \mathbf{u} d y .\right.
\end{aligned}
$$

Since $t_{1}, \ldots, t_{n} \in E$ are distinct, (3.10) and (ii) of condition (C2) imply that the Gaussian random variables $X_{k}\left(t_{j}\right)(k=1, \ldots, d, j=1, \ldots, n)$ are linearly independent. Hence by applying generalized Hölder's inequality, and 
Lemmas 3.4 and 3.5, we derive that $\mathcal{M}_{n}$ is bounded by a constant multiple of

$$
\begin{aligned}
& \prod_{j=1}^{n}\left\{\int_{\mathbb{R}} \int_{\mathbb{R}^{n d}}\left[\frac{\left|\exp \left(i u_{j}^{k_{j}} y^{k_{j}}\right)-1\right|}{p\left(\left|y^{k_{j}}\right| / \sqrt{d}\right)}\right]^{n}\right. \\
& \left.\times \exp \left[-\frac{1}{2} \operatorname{Var}\left(\sum_{j=1}^{n} \sum_{l=1}^{d} u_{j}^{l} X_{l}\left(t_{j}\right)\right)\right] d \mathbf{u} d y^{k_{j}}\right\}^{1 / n}
\end{aligned}
$$

$$
\begin{aligned}
= & \frac{(2 \pi)^{n(d-1) / 2}}{\left[\operatorname{det} \operatorname{Cov}\left(Y\left(t_{1}\right), \ldots, Y\left(t_{n}\right)\right)\right]^{d / 2}} \\
& \times \prod_{j=1}^{n}\left\{\int_{\mathbb{R} \mathbb{R}} \frac{\left|\exp \left(i u_{j}^{k_{j}} y^{k_{j}} / \sigma_{j}\right)-1\right|^{n}}{p^{n}\left(\left|y^{k_{j}}\right| / \sqrt{d}\right)} \exp \left(-\left(u_{j}^{k_{j}}\right)^{2} / 2\right) d y^{k_{j}} d u_{j}^{k_{j}}\right\}^{1 / n} \\
\leq & \frac{K_{3,8}^{n}}{\left[\operatorname{det} \operatorname{Cov}\left(Y\left(t_{1}\right), \ldots, Y\left(t_{n}\right)\right)\right]^{d / 2}} \prod_{j=1}^{n}\left[\int_{0}^{\infty} p_{+}^{-n}\left(\sigma_{j} / v \sqrt{d}\right) \exp \left(-v^{2} / 2\right) d v\right]^{1 / n},
\end{aligned}
$$

where $K_{3,8}>0$ is a constant depending on $D$ and $K_{3,5}$ in Lemma 3.5, and $\sigma_{j}^{2}$ is the conditional variance of $X_{k_{j}}\left(t_{j}\right)$ given $X_{l}\left(t_{i}\right)\left(l \neq k_{j}\right.$ or $l=k_{j}$, $i \neq j)$.

Since

$$
p_{+}^{-n}(x / \sqrt{d})= \begin{cases}\log ^{n \gamma}(e / x) & \text { if } 0<x<1 \\ 1 & \text { if } x \geq 1\end{cases}
$$

and $\log _{+}^{\alpha}(x y) \leq 2^{\alpha}\left(\log _{+}^{\alpha} x+\log _{+}^{\alpha} y\right)$ for all $\alpha \geq 0$, where we have written $\log _{+} x=\max \{1, \log x\}$, we deduce

$$
\begin{aligned}
& \int_{0}^{\infty} p_{+}^{-n}\left(\sigma_{j} / v \sqrt{d}\right) \exp \left(-v^{2} / 2\right) d v \\
& \leq \int_{\sigma_{j} / v \geq 1} \exp \left(-v^{2} / 2\right) d v+2^{n \gamma} \int_{\sigma_{j} / v<1} \log _{+}^{n \gamma}(v) \exp \left(-v^{2} / 2\right) d v \\
& \quad+2^{n \gamma} \int_{\sigma_{j} / v<1} \log _{+}^{n \gamma}\left(e / \sigma_{j}\right) \exp \left(-v^{2} / 2\right) d v
\end{aligned}
$$

By Lemma 3.6, for $n$ large enough, the above is bounded by

$$
K^{n}\left[\log _{+}^{n \gamma}\left(e / \sigma_{j}\right)+(\log (n \gamma))^{n \gamma}\right] \leq K_{3,9}^{n}\left[\log _{+}^{n \gamma}\left(e / \sigma_{j}\right)\right](\log n)^{n \gamma} .
$$

It follows from (3.18) and (3.20)-(3.23) that

$$
\begin{aligned}
\mathbb{E} \int_{D} \int_{D}( & \left.\frac{l_{\mu}(x)-l_{\mu}(y)}{p(|x-y| / \sqrt{d})}\right)^{n} d x d y \\
\leq & K_{3,10}^{n} \lambda_{d}(D)(\log n)^{n \gamma} \\
& \quad \int_{E_{\neq}^{n}} \frac{1}{\left[\operatorname{det} \operatorname{Cov}\left(Y\left(t_{1}\right), \ldots, Y\left(t_{n}\right)\right)\right]^{d / 2}} \prod_{j=1}^{n} \log _{+}^{\gamma}\left(\frac{e}{\sigma_{j}}\right) \mu^{n}(d \mathbf{t}) .
\end{aligned}
$$


Using again the independence of $X_{1}, \ldots, X_{d}$ and condition (C2), we deduce

$$
\begin{aligned}
\sigma_{j}^{2} & =\operatorname{Var}\left(X_{k_{j}}\left(t_{j}\right) \mid X_{l}\left(t_{i}\right): l \neq k_{j} \text { or } l=k_{j}, i \neq j\right) \\
& =\operatorname{Var}\left(X_{k_{j}}\left(t_{j}\right) \mid X_{k_{j}}\left(t_{i}\right): i \neq j\right) \\
& \geq K_{3,3} \psi\left(\min \left\{\left|t_{j}-t_{i}\right|: i=0 \text { or } i \neq j\right\}\right) \quad\left(t_{0}=0\right) .
\end{aligned}
$$

Now, for any fixed $\mathbf{t}=\left(t_{1}, \ldots, t_{n}\right) \in E_{\neq}^{n}$, we define a permutation $\pi$ of $\{1, \ldots, n\}$ such that

$$
\begin{aligned}
\left|t_{\pi(1)}\right| & =\min \left\{\left|t_{i}\right|: i=1, \ldots, n\right\}, \\
\left|t_{\pi(j)}-t_{\pi(j-1)}\right| & =\min \left\{\left|t_{i}-t_{\pi(j-1)}\right|:\right. \\
& i \in\{1, \ldots, n\} \backslash\{\pi(1), \ldots, \pi(j-1)\}\} .
\end{aligned}
$$

Notice that the integrand on the right-hand side of (3.24) is permutation invariant in $t_{1}, \ldots, t_{n}$. So by (3.25), (3.26) and the fact that diam $E$ is small, we can write

$$
\begin{aligned}
& \prod_{j=1}^{n} \log _{+}^{\gamma}\left(e / \sigma_{j}\right) \\
& \leq \prod_{j=1}^{n} \log _{+}^{\gamma}\left(\frac{K_{3,11}}{\psi\left(\min \left\{\left|t_{\pi(j)}-t_{i}\right|: i=0 \text { or } i \neq \pi(j)\right\}\right)}\right) \\
& \leq K_{3,12}^{n} \prod_{j=1}^{n} \log _{+}^{\gamma}\left(\frac{1}{\psi\left(\min \left\{\left|t_{\pi(j)}-t_{\pi(j-1)}\right|,\left|t_{\pi(j+1)}-t_{\pi(j)}\right|\right\}\right)}\right) \\
& \leq K_{3,12}^{n} \prod_{j=1}^{n} \log _{+}^{2 \gamma}\left(\frac{1}{\psi\left(\left|t_{\pi(j)}-t_{\pi(j-1)}\right|\right)}\right) .
\end{aligned}
$$

Combining (3.10) and (3.27), we obtain

$$
\begin{aligned}
& \frac{1}{\left[\operatorname{det} \operatorname{Cov}\left(Y\left(t^{1}\right), \ldots, Y\left(t^{n}\right)\right)\right]^{d / 2}} \prod_{j=1}^{n} \log _{+}^{\gamma}\left(e / \sigma_{j}\right) \\
& \leq K_{3,13}^{n} \prod_{j=1}^{n} \frac{1}{\psi\left(\left|t_{\pi(j)}-t_{\pi(j-1)}\right|\right)^{d / 2}} \log _{+}^{2 \gamma}\left(\frac{1}{\psi\left(\left|t_{\pi(j)}-t_{\pi(j-1)}\right|\right)}\right) .
\end{aligned}
$$

Define $\Gamma_{\pi}=\left\{\mathbf{t} \in E_{\neq}^{n}: \mathbf{t}\right.$ satisfies (3.26) $\}$ and write $\Gamma_{\pi}$ as $\Gamma$ when $\pi$ is the identity permutation. It follows from (3.24), the permutation invariance of the integrand in its right-hand side, (3.28) and (3.5) that 


$$
\begin{aligned}
& \quad \mathbb{E} \int_{D} \int_{D}\left(\frac{l_{\mu}(x)-l_{\mu}(y)}{p(|x-y| / \sqrt{d})}\right)^{n} d x d y \\
& \leq K_{3,14}^{n} n !(\log n)^{n \gamma} \int_{\Gamma} \prod_{j=1}^{n} \frac{1}{\psi\left(\left|t_{j}-t_{j-1}\right|\right)^{d / 2}} \log _{+}^{2 \gamma}\left(\frac{1}{\psi\left(\left|t_{j}-t_{j-1}\right|\right)}\right) \mu^{n}(d \mathbf{t}) \\
& \leq K_{3,6}^{n} n !(\log n)^{n \gamma} .
\end{aligned}
$$

This finishes the proof of Lemma 3.8.

REMARK 3.9. Let $X_{1}, \ldots, X_{d}$ be independent Gaussian random fields such that for each $j=1, \ldots, d, X_{j}$ satisfies condition (C2) for some function $\psi_{j}$. Then the $(N, d)$-Gaussian field $X=\left\{X(t), t \in \mathbb{R}^{N}\right\}$ defined by $X(t)=\left(X_{1}(t), \ldots, X_{d}(t)\right)$ is anisotropic in $\mathbb{R}^{d}$. Theorem 3.2 can be extended to such $(N, d)$-Gaussian random fields. We leave the details to the interested reader.

QUESTION 3.10. We have mentioned that, if $E \subset \mathbb{R}^{N}$ carries a probability measure $\mu$ such that (3.4) holds (i.e., $\operatorname{Cap}_{\psi^{-d / 2}}(E)>0$ ), then $X(E)$ a.s. has positive Lebesgue measure. It is an open problem to find a necessary and sufficient condition on $E$ for $\mathbb{E}\left[\lambda_{d}(X(E))\right]>0$. This problem has a long history and is closely related to potential theory of Gaussian random fields; see Khoshnevisan [20] for more information. For $(N, d)$-Gaussian random fields, the only known result is the following due to Khoshnevisan [19] for $N=2$ and Khoshnevisan and Xiao [21] for general $N$ : If $W=\left\{W(t), t \in \mathbb{R}_{+}^{N}\right\}$ is the Brownian sheet (or an additive Brownian motion) in $\mathbb{R}^{d}$ and $E \subset \mathbb{R}_{+}^{N}$ is a Borel set, then $\mathbb{E}\left[\lambda_{d}(W(E))\right]>0$ if and only if $\operatorname{Cap}_{d / 2}(E)>0$.

Acknowledgements. The work of this paper was done during the second author's visit to the Mathematics Division of the National Center for Theoretical Sciences at Taipei. He thanks the hospitality of his host. The authors thank the referee for his/her comments which have led to several improvements of the manuscript.

\section{References}

[1] R. J. Adler, The Geometry of Random Fields, Wiley, New York, 1981.

[2] V. V. Anh, J. M. Angulo and M. D. Ruiz-Medina, Possible long-range dependence in fractional random fields, J. Statist. Plann. Inference 80 (1999), 95-110.

[3] S. M. Berman, Gaussian processes with biconvex covariances, J. Multivariate Anal. 8 (1978), 30-44.

[4] -, Spectral conditions for local nondeterminism, Stochastic Process. Appl. 27 (1987), 73-84.

[5] N. H. Bingham, C. M. Goldie and J. L. Teugels, Regular Variation, Cambridge Univ. Press, Cambridge, 1987.

[6] C. Bluhm, Random recursive construction of Salem sets, Ark. Mat. 34 (1996), 51-63. 
[7] C. Bluhm, On a theorem of Kaufman: Cantor-type construction of linear fractal Salem sets, ibid. 36 (1998), 307-316.

[8] —, Fourier asymptotics of statistically self-similar measures, J. Fourier Anal. Appl. 5 (1999), 355-362.

[9] J. Cuzick and J. P. DuPreez, Joint continuity of Gaussian local times, Ann. Probab. 10 (1982), 810-817.

[10] A. M. Garsia, Continuity properties of Gaussian processes with multidimensional time parameter, in: Proc. 6th Berkeley Sympos. on Math. Statist. and Probability (Univ. of California, Berkeley, CA, 1970/1971), Vol. II, Univ. of California Press, Berkeley, CA, 1972, 369-374.

[11] D. Geman and J. Horowitz, Occupation densities, Ann. Probab. 8 (1980), 1-67.

[12] J.-P. Kahane, Some Random Series of Functions, 2nd ed., Cambridge Univ. Press, Cambridge, 1985.

[13] —, Ensembles aléatoires et dimensions, in: Recent Progress in Fourier Analysis (El Escorial, 1983), North-Holland, Amsterdam, 1985, 65-121.

[14] _, Fractals and random measures, Bull. Sci. Math. 117 (1993), 153-159.

[15] J.-P. Kahane et R. Salem, Ensembles parfaits et séries trigonométriques, 2ème éd., Hermann, Paris, 1994.

[16] Y. Kasahara, N. Kôno and T. Ogawa, On tail probability of local times of Gaussian processes, Stochastic Process. Appl. 82 (1999), 15-21.

[17] R. Kaufman, Fourier analysis and paths of Brownian motion, Bull. Soc. Math. France 103 (1975), 427-432.

[18] -, On the theorem of Jarnik and Besicovitch, Acta Arith. 39 (1981), 265-267.

[19] D. Khoshnevisan, Brownian sheet images and Bessel-Riesz capacity, Trans. Amer. Math. Soc. 351 (1999), 2607-2622.

[20] —, Multiparameter Processes: An Introduction to Random Fields, Springer, New York, 2002.

[21] D. Khoshnevisan and Y. Xiao, Images of the Brownian sheet, Trans. Amer. Math. Soc., to appear.

[22] G. Mockenhaupt, Salem sets and restriction properties of Fourier transforms, Geom. Funct. Anal. 10 (2000), 1579-1587.

[23] E. J. G. Pitman, On the behaviour of the characteristic function of a probability distribution in the neighbourhood of the origin, J. Austral. Math. Soc. Ser. A 8 (1968), 423-443.

[24] L. D. Pitt, Local times for Gaussian vector fields, Indiana Univ. Math. J. 27 (1978), 309-330.

[25] M. Talagrand, Hausdorff measure of trajectories of multiparameter fractional Brownian motion, Ann. Probab. 23 (1995), 767-775.

[26] —, Multiple points of trajectories of multiparameter fractional Brownian motion, Probab. Theory Related Fields 112 (1998), 545-563.

[27] D. Wu and Y. Xiao, Geometric properties of the images of fractional Brownian sheets, submitted, 2006.

[28] Y. Xiao, Hausdorff measure of the sample paths of Gaussian random fields, Osaka J. Math. 33 (1996), 895-913.

[29] —, Hölder conditions for the local times and the Hausdorff measure of the level sets of Gaussian random fields, Probab. Theory Related Fields 109 (1997), 129-157.

[30] - , The packing measure of the trajectories of multiparameter fractional Brownian motion, Math. Proc. Cambridge Philos. Soc. 135 (2003), 349-375.

[31] _ Strong local nondeterminism and sample path properties of Gaussian random fields, submitted, 2005. 
[32] Y. Xiao and T. Zhang, Local times of fractional Brownian sheets, Probab. Theory Related Fields 124 (2002), 204-226.

[33] A. M. Yaglom, Some classes of random fields in $n$-dimensional space, related to stationary random processes, Teor. Veroyatnost. i Primenen. 2 (1957), 292-338 (in Russian); English transl.: Theory Probab. Appl. 2 (1957), 273-320.

[34] A. Zygmund, Trigonometric Series, Vol. I, Cambridge Univ. Press, Cambridge, 1959.

Department of Mathematics

National Taiwan University

Taipei 10617, Taiwan

E-mail: shiehnr@math.ntu.edu.tw
Department of Statistics and Probability Michigan State University A-413 Wells Hall East Lansing, MI 48824, U.S.A. E-mail: xiao@stt.msu.edu Web: http://www.stt.msu.edu/ ${ }^{\sim}$ xiaoyimi

Received March 4, 2005

Revised version June 9, 2006 